\title{
Avaliação formativa da aprendizagem com instrumentação em Jogos digitais: Proposta de um framework conceitual
}

\author{
Glauber G. de Araujo ${ }^{1}$, Eduardo Henrique da S. Aranha ${ }^{1}$ \\ ${ }^{1}$ Departamento de Informática e Matemática Aplicada (DIMAp) - Universidade Federal \\ do Rio Grande do Norte (UFRN) \\ Avenida Senador Salgado Filho, 3000 - 59072-970 - Natal, RN - Brasil \\ glauber.galvao@gmail.com, eduardoaranha@dimap.ufrn
}

\begin{abstract}
This paper presents a proposal, with an example of practical application of formative assessment instrumentation of learning in games, as an effort to discuss solutions to the effective use of this assessment in the everyday of learning environments. Formative assessment is a process that verifies that the pedagogical practices produced results in time to remedy students' possible learning disabilities. However, some teachers under the argument of complexity and laborious, reject this evaluation function. In addition, the conventional instrumentation becomes an obstacle with the tedious forms and difficult logistics.
\end{abstract}

Resumo. Este artigo apresenta uma proposta, com um exemplo de aplicação prática, de instrumentação da avaliação formativa da aprendizagem em jogos digitais, como esforço de discutir soluções para o uso efetivo dessa avaliação no cotidiano dos ambientes de aprendizagem. A avaliação formativa é um processo que verifica se as ações pedagógicas produziram resultados nos alunos em tempo de sanar possíveis deficiências de aprendizagem. Entretanto, seu uso frequente é rejeitado por muitos professores sob o argumento de serem complexas e trabalhosas. Além disso, sua instrumentação convencional tornase um obstáculo com seus formulários maçantes e de logística difícil.

\section{Introdução}

O processo de avaliação no contexto da educação precisa ocorrer em conjunto com ações capazes de garantir que o aprendiz receba o apoio do professor para sanar possíveis deficiências de aprendizagem ainda durante o processo de ensino [Perrenoud, 1999].

Dados coletados por meio de avaliações contínuas e sistemáticas, como as que têm função formativa, se concentram principalmente na melhoria do processo ensinoaprendizagem subsidiando o professor com informações para sua regulação [Jorba e Sanmarti, 2003].

Avaliar contínua e sistematicamente durante todo o processo ensinoaprendizagem não é uma tarefa fácil, ainda mais avaliar individualmente os aprendizes em meio a turmas grandes e heterogêneas. Diante dessa dificuldade, a grande maioria dos professores preferem as avaliações que classificam os alunos por conceito depois do processo de ensino [Hadji, 2001].

Sob outro ângulo, faltam aos professores recursos pedagógicos suficientemente diversificados que os auxiliem na geração de dados e tempo para interpretá-los, como 
também a realização das intervenções regulatórias no momento apropriado [Perrenoud, 1999; Quinquer, 2003].

A instrumentação convencional das avaliações formativas é também um entrave à sua prática. Entende-se por instrumentação todos os meios que geram dados de conhecimento do aprendiz, como provas, relatórios e questionários. O processo realizado com questionários impressos, por exemplo, aplicados várias vezes ao longo do período letivo, são maçantes para os aprendizes e têm uma logística difícil para o professor.

Essa problemática motivou o desenvolvimento de um framework conceitual, no domínio da avaliação da aprendizagem, para apoiar a avaliação formativa da aquisição de conhecimento com sua instrumentação em jogos digitais.

O framework, ora proposto, une a instrumentação da avaliação formativa com o ambiente computacional e os elementos lúdicos presentes nos jogos digitais. A finalidade é tornar a tarefa de avaliar a aquisição de conhecimento em áreas diversas mais estimulante para o aprendiz e mais factível para o professor.

Mais estimulante porque permite a imersão dos alunos em jogos que oferecem desafios, benefícios, recompensas e o ranking que os classificam diante do grupo. É mais factível para o professor por duas razões: primeiro porque não baseia a avaliação cotidiana somente em aspectos intuitivos, frequentes na observação convencional. Segundo porque as tarefas mais exaustivas do processo (consolidação e correção de dados, etc.) são executadas em ambiente computacional.

Nesse sentido, espera-se que o framework contribua no processo ensinoaprendizagem, auxiliando professores na atividade de avaliar continuamente e alunos na obtenção de resultados mais coerentes com os seus desempenhos. Mas, não só isso, espera-se ainda que o jogo digital proporcione um ambiente de avaliação mais lúdico, e desse modo, menos cansativo para quem aplica e para quem é avaliado.

\section{Referencial teórico}

Nesta seção são apresentados os fundamentos teóricos que norteiam a pesquisa e contribuem para a melhor compreensão do contexto onde se insere este artigo.

\subsection{Avaliação da aprendizagem}

Para avaliar é necessário ter clareza do que fazer com as informações que são coletadas no processo, fazendo da avaliação e da tomada de decisões a partir dos resultados, questões-chave para melhorar o conhecimento dos aprendizes [Parcerisa, 2003].

Embora a avaliação ainda seja amplamente utilizada apenas como instrumento de medição para seleção e classificação, crescem as concepções que defendem outra lógica da avaliação, e a concebem com a finalidade de "ajudar a melhorar o processo de aprendizagem dos alunos e ser útil para aprimorar o ensino" [Parcerisa, 2003].

Nesse sentido, Jorba e Sanmarti (2003) defendem que a avaliação tem duas funções básicas: (a) uma de seleção e classificação, que ocorre depois do ensino, conhecida como somativa; (b) outra de ajuste do processo de ensino-aprendizagem, de reconhecimento das mudanças, representadas por duas avaliações conhecidas por diagnóstica e formativa e que ocorrem antes e durante o ensino, respectivamente. 
A avaliação formativa da aprendizagem gera dados úteis para a adequação das atividades de ensino-aprendizagem às necessidades dos aprendizes [Jorba; Sanmarti, 2003] e, dessa maneira, melhora a qualidade do ensino, aproximando-se mais das necessidades de cada aluno, considerando a sua singularidade, procurando superar a perspectiva de um modelo de ensino "tamanho único" que é demais para alguns e insuficiente para outros.

\subsection{Jogos digitais e aprendizagem}

As concepções sobre jogos são várias e abordam desde elementos de entretenimento, passando por elementos pedagógicos até elementos culturais.

A história da contribuição dos jogos nas outras áreas do conhecimento é longa e continua com maior ênfase com os jogos digitais suportados pelas novas tecnologias, não só com o apoio lúdico, como também com o apoio computacional.

Nesse sentido, jogos digitais podem ser mediadores de aprendizagem e podem contribuir para o despertar de competências, habilidades e melhor compreensão dos conteúdos em diversas áreas. Para Huizinga (2000), "todo jogo é capaz, a qualquer momento, de absorver inteiramente o jogador" em uma espécie de imersão, o que Caillois (1990) corrobora afirmando que "o jogo é essencialmente uma ocupação separada, cuidadosamente isolada do resto da existência".

Jogar desenvolve a capacidade de observar regras, seguir procedimentos sistemáticos com disciplina, características que são fundamentais nas práticas científicas. A atenção e o raciocínio lógico, principalmente em jogos de estratégia, são também elementos estimulados com o uso de jogos. O exercício da atenção provoca a imersão do jogador e um estado de extrema concentração na tarefa que está desempenhando.

\subsection{Avaliação formativa e jogos digitais}

Uma revisão sistemática foi realizada no sentido de localizar trabalhos que tratassem especificamente sobre avaliação formativa e jogos digitais. Um total de vinte e cinco trabalhos entre artigos e iniciativas foram encontrados com títulos apontando nessa direção. Contudo, quando os resumos foram lidos, apenas três artigos foram selecionados como relevantes [Araujo, 2013] e são apresentados a seguir:

O trabalho desenvolvido por Cuomo (2011) "Full Formative Assessment based on Educacional Video Games", trata de uma portal na internet (PlayLearn) para exploração de jogos educativos a longo prazo e testes online. O portal registra os parâmetros de aprendizagem para análise em tempo real pelos professores, com o objetivo de melhorar o processo de aprendizagem. O jogo é baseado na "jornada do herói", no qual o personagem do jogador deve recolher diamantes ao longo da jornada enquanto responde a questões sobre o conteúdo estudado.

Em "Web-based quiz-game-like formative assessment: Development and evaluation" [Wang, 2007], é apresentado um sistema de múltipla escolha baseada em Web quiz-jogo como avaliação formativa chamado de GAM-WATA. A pesquisa envolvendo 165 alunos do ensino fundamental em Taiwan central comparou a eficácia de três diferentes tipos de avaliação formativa em um ambiente de e-Learning: teste de papel e lápis (PPT), teste normal baseado na Web (N-WBT) e GAM-WATA. A partir dos resultados, não há nenhuma diferença significativa entre o grupo PPT e o grupo N-WBT 
quando comparada a eficácia do estudante nos quatro conceitos de aprendizagem avaliados. No entanto, os estudantes do grupo GAM-WATA tiveram uma eficácia melhor do que aqueles do grupo PPT. Nesse sentido, o autor indica que o uso de jogos no processo de avaliação formativa motiva mais os alunos á participação do que no PPT ou N-WBT.

Thiry et al. (2010), em seu trabalho "Promovendo a Aprendizagem de Engenharia de Requisitos de Software Através de um Jogo Educativo", apresenta uma forma de avaliar alunos de engenharia de requisitos utilizando o jogo educativo ilha dos requisitos. O objetivo do jogo é ajudar o personagem, a fugir da ilha dos requisitos - uma ilha habitada por tribos canibais que tem um vulcão prestes a entrar em erupção. $O$ jogador deve enfrentar uma série de desafios relacionados ao conteúdo da disciplina de engenharia de requisitos. Os autores concluíram que a maioria dos alunos ficaram motivados e gostaram de jogar o jogo, considerando-o relevante para o aprendizado e que pode ser efetivo para ensinar Engenharia de Requisitos nos níveis de entendimento e compreensão.

Os artigos analisados tratam de avaliação formativa utilizando jogos, mas não definem um método sistemático que qualifica e mede a aquisição de conhecimento dos alunos de modo a subsidiar as ações do professor. É nesse ponto que o framework, ora proposto, pode contribuir com essa discussão de um modo efetivo.

\section{Framework conceitual de avaliação formativa: Definição e Aplicação}

Nesta seção, é apresentado o framework conceitual proposto por este trabalho, começando pela metodologia empregada para o seu desenvolvimento e depois pelo detalhamento de seus componentes. Concomitante a apresentação, são descritas as etapas de um exemplo de aplicação prática de utilização do framework em sala de aula.

\subsection{Metodologia empregada}

Com base nos estudos de IHC (Interação Humano Computador), um framework conceitual é um conjunto de ideias e conceitos que se inter-relacionam para descrever como um sistema deve se comportar, se parecer e ser compreendido pelos usuários da maneira pretendida [Preece et al., 2005].

Nesse contexto, a elaboração do framework conceitual, aqui proposto, baseou-se nas ideias de Castillo et al. (2011) acerca da avaliação da aprendizagem, que sugerem a exploração de algumas técnicas da observação sistemática, como apoio na tutoria de alunos. Igualmente, foi considerada a proposta de Jorba e Sanmarti (2003) de uma avaliação em três etapas: coleta de dados, análise dos dados e tomada de decisão.

De maneira geral, o objetivo principal que guiou o desenvolvimento do framework, foi o de conseguir apoiar com ele a instrumentação da avaliação formativa no ambiente computacional do jogo digital. A função do framework é a de sistematizar o processo de avaliação de modo que respostas, competências e habilidades dos alunos sejam coletadas e analisadas para subsidiar as ações pedagógicas do professor.

Para isso, o framework foi estruturado com quatro componentes que desempenham funções específicas e que se inter-relacionam de modo cíclico e contínuo: (a) instrumentar; (b) coletar; (c) analisar e; (d) regular, conforme são descritos a seguir. 


\subsection{Instrumentar}

Instrumentar é o componente que consiste na forma do questionamento aos aprendizes e do registro das respostas. Pode explorar os vários tipos de instrumentos avaliativos conhecidos que se adaptam às situações de ensino e de aprendizagem propostas pelo professor, como também pode explorar os vários gêneros de jogos digitais como instrumentos de verificação de competências e habilidades.

A forma de questionamento pode ser explícita ou tácita. A primeira estimula respostas diretas do aprendiz por meio de questões acerca do conteúdo estudado. Consiste de alguns tipos de instrumentações convencionais, como provas e questionários.

A segunda forma captura "respostas" sem que o aluno seja questionado diretamente sobre o conteúdo estudado. Consiste no uso de alguns gêneros de jogos (por exemplo: Casuais, Simulação, Esportes, Adventure, RPG, Estratégia, Puzzle) que estimulam a prática de habilidades capazes de expressar competências para a solução de desafios nos domínios da linguagem, da matemática e dos fenômenos naturais, bem como o enfrentamento de situações-problema e a construção de argumentações consistentes.

\section{Exemplo de aplicação prática}

Para exemplificar a aplicação do framework apoiando a instrumentação de avaliação formativa em um jogo digital, começando pelo componente Instrumentar, considere a disciplina de Redes de Computadores "Administração de Redes em Software Proprietário", na qual são estudados os conteúdos conforme os módulos: (a) Introdução; (b) Administração do Sistema; (c) Gerenciamento de Serviços e; (d) Núcleo do Sistema.

No seu planejamento, o professor definiu para o módulo "Administração de Sistemas" dois objetivos para a aula sobre o "Agendador de tarefas do Windows Server 2008”. O aluno deverá ser capaz de: (Objetivo 1) Reconhecer as ferramentas de apoio nas atividades assíncronas ou processos interdependentes e; (Objetivo 2) Implantar e configurar essas ferramentas em laboratório.

Cinco atividades formativas (A1 até A5), que avaliam a aquisição do conhecimento na disciplina, foram planejadas contendo dez questões (Q1 até Q10) cada uma, abordando os conceitos-chave nominados de $\alpha$ (alfa), $\beta$ (beta), $\gamma$ (gama) e $\delta$ (delta) repetidamente em todas as atividades.

No processo de execução do planejamento, durante as aulas expositivas, o professor abordou os conceitos-chave propostos para a disciplina, dentre eles, o conceito "automação de atividades rotineiras pelo sistema operacional" que será chamado daqui em diante de conceito $\beta$ (beta). Apresentou também as "ferramentas de monitoramento, gerenciamento de tarefas e ação efetiva" relativas ao conceito.

O professor cadastrou na área de gerenciamento do jogo digital, que deverá utilizar para a instrumentação da avaliação, uma forma explícita de questionamento, o instrumento "Questionário", com as atividades avaliativas planejadas (A1 até A5) que abrangem os conceitos-chave trabalhados na disciplina.

As questões elaboradas para o instrumento "questionário" sugerem respostas dicotômicas (concordo ou discordo), com possibilidade de abstenção (não sei). O acerto 
é valorado com 1 (um), o erro com 0 (zero) e a abstenção não tem valor. O quadro da Figura 1 exibe o exemplo de uma questão.

O snap-in MMC do Agendador de Tarefas do Windows Server 2008 permite que se agende tarefas automatizadas que realizem ações em um horário específico ou quando um determinado evento ocorrer. É correto afirmar que os seus dois principais conceitos são os disparadores e as ações.

[concordo]

[discordo]

[não sei]

Figura 1 - Exemplo de questão do tipo dicotômica

Após a resposta do aluno, o instrumento suplementa o enunciado com informações que aprofundam os conceitos apresentados, com o objetivo de melhorar o nível de conhecimento do aluno, seja a resposta errada ou correta ou com abstenção. As respostas ás questões e o escore de desempenho no jogo são registrados concomitantemente, mas com finalidades distintas. As respostas são coletadas para análise pedagógica e os escores classificam o aluno em ranking de desempenho entre todos os jogadores.

\subsection{Coletar}

Coletar é o componente que consiste na captura das respostas registradas pelo instrumento de avaliação. A coleta é realizada mediante o atendimento a critérios que expressam os objetivos almejados pelo professor e as respostas coletadas precisam ser valoradas segundo indicadores quantificadores que expressam os critérios.

O processo inicia pela definição da relação entre as capacitações do domínio do conhecimento e do domínio dos processos cognitivos, conforme a taxonomia de Bloom revisada [Anderson et al., 2001], chamada de critério formativo [Araujo, 2013].

O critério formativo deve expressar qualitativamente os objetivos pedagógicos almejados pelo professor em acordo com as capacitações da taxonomia empregada.

A segunda etapa do processo é a definição dos indicadores formativos que quantificam os critérios formativos emitidos e os tornam mensuráveis para que sejam analisados por métricas de verificação de aprendizagem [Araujo, 2013].

\section{Exemplo de aplicação prática}

Retomando o exemplo proposto, os critérios formativos que atendem aos objetivos definidos pelo professor no planejamento são identificados na tabela Dimensão do Conhecimento X Dimensão dos Processos Cognitivos, por meio da indicação das duas dimensões que estabelecem a relação linha-coluna [Anderson et al., 2001].

$\mathrm{Na}$ tabela a seguir foram identificadas as relações "B1" (que corresponde ao critério Conceitual-Lembrar e atende ao Objetivo 1) e "C3" (que corresponde ao critério Procedimental-Aplicar e atende ao Objetivo 2).

Tabela 1 - Seleção das capacitações com a definição da relação linha-coluna

\begin{tabular}{|l|c|c|c|c|c|c|}
\hline \multirow{2}{*}{$\begin{array}{c}\text { DIMENSÃo Do } \\
\text { CONHECIMENTO }\end{array}$} & $\begin{array}{c}\text { DIMENSÃ DOS PROCESSOS COGNIVOS } \\
\text { Lembrar }\end{array}$ & $\begin{array}{c}(2) \\
\text { Entender }\end{array}$ & $\begin{array}{c}(3) \\
\text { Aplicar }\end{array}$ & $\begin{array}{c}\text { (4) } \\
\text { Analisar }\end{array}$ & $\begin{array}{c}\text { (5) } \\
\text { Avaliar }\end{array}$ & $\begin{array}{c}(6) \\
\text { Criar }\end{array}$ \\
\hline (A) Factual & & & & & & \\
\hline (B) Conceitual & Objetivo 1 & & & & & \\
\hline (C) Procedimental & & & Objetivo 2 & & & \\
\hline (D) Metacognitivo & & & & & & \\
\hline
\end{tabular}


O resultado da definição dos critérios formativos é a emissão das expectativas de aprendizagem das dimensões combinadas, ou seja, dos critérios formativos identificados que atendam aos objetivos 1 e 2 do exemplo. Nesse caso, espera-se que o aprendiz seja capaz, respectivamente, de:

(1) estabelecer relações entre as ferramentas de apoio nas atividades assíncronas ou processos interdependentes e entre suas partes que as fazem funcionar em conjunto (conceitual) por meio do domínio sobre a produção da informação certa a partir da memória (lembrar) e;

(2) Implantar e configurar as ferramentas de apoio nas atividades assíncronas ou processos interdependentes em laboratório (aplicar) orientado pelos critérios para determinar quando usar os procedimentos apropriados (procedimental).

Esses critérios formativos emitidos precisam agora ser quantificados para serem medidos. O método de escolha dos indicadores formativos definido em [Araujo, 2013] define os indicadores que serão utilizados na tarefa de quantificar os critérios formativos. Para essa simulação foi selecionado o indicador "número de acertos" das respostas para quantificar o "critério formativo 1".

\subsection{Analisar}

O componente analisar verifica os indicadores formativos, por meio da aplicação de métricas próprias, com a finalidade de identificar se eles expressam e como expressam a aquisição de conhecimento dos alunos nas atividades desempenhadas.

As métricas utilizadas devem ser capazes de medir os indicadores formativos e demonstrar resultados legíveis para o professor, como por exemplo, a evolução da aquisição de conhecimento dos aprendizes nos conceitos-chave ao longo de um período.

\section{Exemplo de aplicação prática}

Retornando ao exemplo, a análise dos indicadores que quantificam o "critério formativo 1" pode ser realizada através da métrica ACC (Aquisição de Conceitos-Chave) adaptada a partir de [Pimentel, 2006] para analisar a aquisição de conceitos-chave estudados em um período de tempo e expressar a evolução da aprendizagem do aluno. A ACC é calculada a partir da média ponderada das medidas de desempenho obtidas pelos aluno nas atividades executadas.

Os passos a seguir demonstram a definição e análise da ACC de dois alunos nas cinco atividades (A1 até A5) que contemplam repetidamente o conceito $\beta$ (beta), que corresponde a "automação de atividades rotineiras pelo sistema operacional".

O objetivo é medir os indicadores formativos (número de acertos), analisá-los e, conhecer o quanto sabe os alunos, individualmente e em comparação com a média da turma.

Passo 1: O número de acertos alcançado por cada aluno nas atividades, daqui em diante chamado de "medidas de desempenho", são tabulados na matriz Alunos X Atividades. Os pesos definidos $(\mathrm{A} 1=1, \mathrm{~A} 2=2, \mathrm{~A} 3=3, \mathrm{~A} 4=4$ e $\mathrm{A} 5=5)$ são fundamentais para definir $\mathrm{o}$ grau de importância da atividade. 
Tabela 2 - Medidas de desempenho com seus pesos

\begin{tabular}{|c|r|r|r|r|r|}
\hline Pesos das atividades & \multicolumn{1}{|l|}{2} & \multicolumn{1}{|c|}{3} & \multicolumn{1}{|c|}{5} \\
\hline ALUNO A & 2 & 5 & 4 & 4 & 5 \\
\hline ALUNO B & 7 & 8 & 9 & 9 & 10 \\
\hline
\end{tabular}

Passo 2: As medidas de desempenho das atividades são multiplicadas por $10^{-1} \mathrm{com} o$ objetivo de converter os valores em índices decimais.

Tabela 3 - Medidas de desempenho convertidas

\begin{tabular}{|c|c|c|c|c|c|}
\hline Pesos das atividades & 1 & 2 & 3 & 4 & 5 \\
\hline Alunos & A1 & A2 & A3 & A4 & A5 \\
\hline ALUNO A & 0,20 & 0,50 & 0,40 & 0,40 & 0,50 \\
\hline ALUNO B & 0,70 & 0,80 & 0,90 & 0,90 & 1,00 \\
\hline
\end{tabular}

Passo 3: Baseado nos índices e pesos do passo anterior, a ACC de cada atividade, é calculada por meio de média ponderada considerando para o cálculo na atividade atual, os valores das atividades anteriores, conforme demonstrado nas seguintes fórmulas:

$$
\text { Onde : } A C C(A 1)=\frac{(A 1 \times 1)}{1} \text { até } A C C(A 5)=\frac{(\mathrm{A} 1 \times 1+\mathrm{A} 2 \times 2+\mathrm{A} 3 \times 3+\mathrm{A} 4 \times 4+\mathrm{A} 5 \times 5)}{(1+2+3+4+5)}
$$

Os resultados encontrados são demonstrados na tabela a seguir:

Tabela 4 - Métrica ACC calculada

\begin{tabular}{|l|r|r|r|r|r|}
\hline \multicolumn{1}{|c|}{ Alunos } & A1 & A2 & A3 & A4 & \multicolumn{1}{c|}{ A5 } \\
\hline Média da Turma & 0,60 & 0,50 & 0,70 & 0,60 & 0,80 \\
\hline ALUNO A & 0,20 & 0,40 & 0,40 & 0,40 & 0,43 \\
\hline ALUNO B & 0,70 & 0,77 & 0,83 & 0,86 & 0,91 \\
\hline
\end{tabular}

Através da ACC pode-se verificar por meio de gráficos e mesmo por comparações pontuais a evolução da aquisição do conhecimento individual e em grupo dos alunos naqueles conceitos-chave ao longo do processo ensino-aprendizagem.

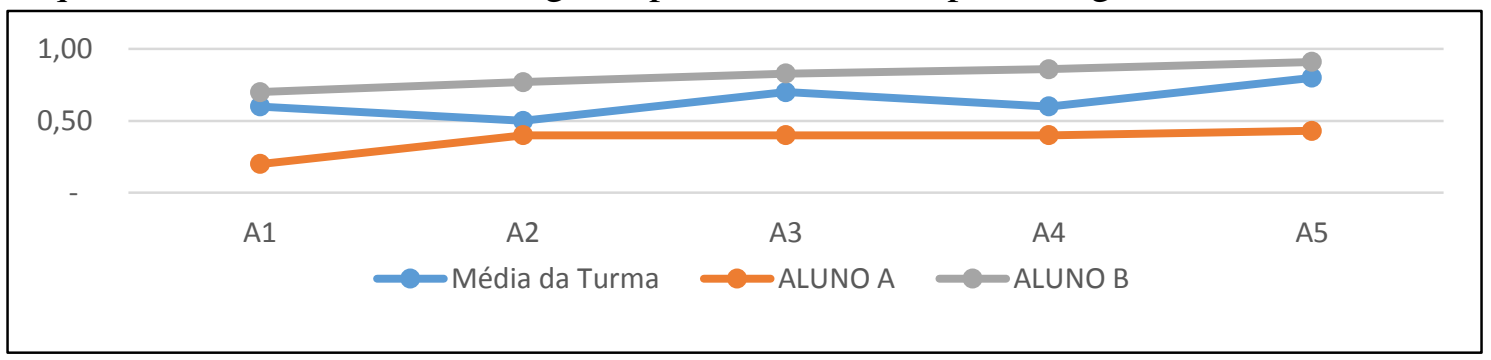

Figura 2 - Gráfico de evolução da ACC

O gráfico demonstra que em comparação aos índices da ACC da turma o "Aluno A" mantém-se abaixo do esperado enquanto o "Aluno B" obteve em todo o tempo um desempenho sempre acima da média de seu grupo.

Contudo, observando os resultados individualmente do "Aluno A" percebe-se uma evolução positiva do desempenho entre as atividades A1 e A2, mantendo-se estável ao longo das demais atividades. Enquanto isso o "Aluno B" desenvolve uma tendência de crescimento desde a primeira atividade até a última.

Essas informações podem suprir o professor com subsídios importantes para suas ações no sentido de sanar possíveis deficiências identificadas. Ações cujos resultados podem ser percebidos, como no intervalo entre as atividades A1 e A2 do "Aluno A", que 
após um baixo desempenho na atividade A1 o aluno recebeu a intervenção individual do professor que reforçou o conceito-chave através de revisão e o melhor resultado já apareceu na atividade seguinte A2, mantendo-se estável até a última atividade.

\subsection{Regular}

A regulação da aprendizagem é desenvolvida pelo professor a partir dos resultados emitidos pelas análises de dados realizadas, com a finalidade de sanar possíveis deficiências detectadas em tempo de processo ensino-aprendizagem. Alguns relatórios orientam as ações do professor (por exemplo: revisões, atendimentos individuais, etc.) no sentido de reverter essas deficiências. As ações pedagógicas ainda durante o processo ensino-aprendizagem é vital para que o aprendiz não chegue ao final do período letivo com acúmulos de dificuldades nos conceitos-chave.

\subsection{Ambiente computacional do Jogo Digital}

O jogo digital é o ambiente computacional que apoia o instrumento de avaliação formativa em suas duas formas de questionamento (explícita e tácita). Fornece a ele não só os recursos computacionais como também o ambiente lúdico para a imersão do aprendiz e permite a coleta de dados da interação do jogador com o jogo e do desempenho do aprendiz nas soluções dadas nas formas de questionamentos.

Em tese, qualquer gênero de jogo digital pode ser utilizado para instrumentação de avaliações formativas. O framework pode orientar a concepção de jogos digitais quando incluído no seu projeto de desenvolvimento. O modo como isso é feito foi abstraído deste artigo para dá mais espaço à definição do framework.

\section{Considerações finais}

A pesquisa ora apresentada desenvolve-se na interface entre ciência da computação e a educação. Compreende, por um lado, a importância dos jogos digitais no contexto educacional, tendo em vista a estreita relação dos aprendizes com o universo das tecnologias da informação e da comunicação na atualidade. Por outro, evidencia as dificuldades em se avaliar continuamente o conhecimento, considerando o universo complexo, amplo e heterogêneo de alunos, com os quais os educadores têm que lidar cotidianamente.

Nesse sentido, o presente artigo destaca um aspecto da pesquisa cujo intuito contribuir em duas dimensões da educação. A primeira é para o trabalho de avaliação do professor, a fim de que o processo ensino-aprendizagem obtenha resultados mais coerentes com os seus propósitos iniciais. A segunda é para o ambiente de avaliação do aluno, a fim de que se torne mais lúdico, e desse modo, menos enfadonho, diminuindo as frequentes queixas dos alunos sobre avaliações cansativas.

É clara a complexidade do ato de avaliar continuamente, com base nos pesquisadores da área da educação. Sendo assim, o enfrentamento de um aspecto tão importante quanto difícil do processo ensino-aprendizagem, é necessário. Para tanto, a pesquisa envolveu a elaboração de um framework conceitual que propõe um modelo de apoio a avaliação formativa instrumentada em jogos digitais.

A intenção é criar jogos digitais apoiados pelo framework, que auxiliem o professor a melhorar a qualidade e o controle do nível de aquisição de conhecimento dos 
alunos, uma vez que será possível mensurar, de forma automatizada, o domínio de aquisição dos conceitos-chave que ele, professor, definiu como importante para os seus aprendizes internalizarem.

\section{Referências Bibliográficas}

ANDERSON et al. A Taxonomy for Learning, Teaching and Assessing: a revision of Bloom's taxonomy of educational objectives. New York: Longman, 2001.

ARAUJO, Glauber G. Um Framework Conceitual para apoiar a instrumentação da Avaliação Formativa da Aprendizagem em Jogos Digitais. 2013. Dissertação de Mestrado - Universidade Federal do Rio Grande do Norte, Natal.

CAILLOIS, Roger. Os jogos e os Homens. trad. José G. Palha. Lisboa: Cotovia, 1990.

CASTILlO ARREDONDO, Santiago; GONZÁLES, Luis Polanco; GONZÁLES, Jose Antonio Torres. Formação de tutores: fundamentos teóricos e práticos. Tradução Sandra Martha Dolinsky. Ibpex. Curitiba. 2011.

CUOMO, Salvatore et al. Full Formative Assessment based on Educacional Video Games. 2011. Disponível em: http://weblab.dmi.unisa.it/. Acesso em: 26/01/2013.

HADJI,C. Avaliação desmistificada. Porto Alegre: Artes Médicas, 2001.

HUIZINGA, Johan. Homo Ludens. trad. João P. Monteiro. São Paulo: Perspectiva, 2000.

JORBA, Jaume; SANMARTÍ, Neus. A função pedagógica da avaliação. In: BALLESTER, Margarida (Org.). Avaliação como apoio à aprendizagem. trad. Valério Campos. Porto Alegre: Artmed, 2003. Pág. 23-45.

PARCERISA, Artur. Introdução. In: BALLESTER, Margarida (Org.). Avaliação como apoio à aprendizagem. trad. Valério Campos. Porto Alegre: Artmed, 2003. Pág. 11.

PERRENOUD, Philippe. Avaliação: da excelência à regulação das aprendizagens - entre duas lógicas. trad. Patrícia Chittoni Ramos. Porto Alegre: Artmed, 1999.

PIMENTEL, Edson Pinheiro. Um Modelo para Avaliação e Acompanhamento Contínuo do Nível de Aquisição de Conhecimento do Aprendiz. 2006. 218f. Tese de Doutorado - Instituto Tecnológico de Aeronáutica, São José dos Campos. Pág. 126-133.

PREECE, J.; ROGERS, I.; SHARP, H. Design de Interação: Além da Interação HumanoComputador; Porto Alegre: Bookman, 2005.

QUINQUER, Dolors. Modelos e enfoques sobre a avaliação: O modelo comunicativo. In: BALLESTER, Margarida (Org.). Avaliação como apoio à aprendizagem. trad. Valério Campos. Porto Alegre: Artmed, 2003. Págs. 15-22.

THIRY, Marcello; ZOUCAS, Alessandra; GONÇALVES. Rafael Queiroz. Promovendo a Aprendizagem de Engenharia de Requisitos de Software Através de um Jogo Educativo. In: SIMPÓSIO BRASILEIRO DE INFORMÁTICA NA EDUCAÇÃO, 21 , 2010, Brasília. XXI Simpósio Brasileiro de Informática na Educação - SBIE. João Pessoa: UFPB, 2010.

WANG, Tzu-Hua. Web-based quiz-game-like formative assessment: Developmet and avaluation. 2007. Disponível em: http://www.sciencedirect.com/science/article/. Acesso em: 26/03/2013. 